\title{
Sea state estimation using multiple ships simultaneously as sailing wave buoys
}

\author{
Nielsen, Ulrik D.; H. Brodtkorb, Astrid; J. Sørensen, Asgeir
}

Published in:

Applied Ocean Research

Link to article, DOI:

10.1016/j.apor.2018.12.004

Publication date:

2019

Document Version

Peer reviewed version

Link back to DTU Orbit

Citation (APA):

Nielsen, U. D., H. Brodtkorb, A., \& J. Sørensen, A. (2019). Sea state estimation using multiple ships simultaneously as sailing wave buoys. Applied Ocean Research, 83, 65-76.

https://doi.org/10.1016/j.apor.2018.12.004

\section{General rights}

Copyright and moral rights for the publications made accessible in the public portal are retained by the authors and/or other copyright owners and it is a condition of accessing publications that users recognise and abide by the legal requirements associated with these rights.

- Users may download and print one copy of any publication from the public portal for the purpose of private study or research.

- You may not further distribute the material or use it for any profit-making activity or commercial gain

- You may freely distribute the URL identifying the publication in the public portal 


\title{
PostPrint
}

\section{Sea state estimation using multiple ships simultaneously as sailing wave buoys}

\author{
Ulrik D. Nielsen ${ }^{\mathrm{a}, \mathrm{b}}$, Astrid H. Brodtkorb ${ }^{\mathrm{b}}$, Asgeir J. Sørensen ${ }^{\mathrm{b}}$ \\ ${ }^{a}$ DTU Mechanical Engineering, Technical University of Denmark, Kgs. Lyngby, Denmark \\ ${ }^{b}$ Centre for Autonomous Marine Operations and Systems, NTNU AMOS, Trondheim, Norway
}

\begin{abstract}
The article presents initial ideas towards a network-based approach for sea state estimation used for marine operations and other maritime applications. In principle, all available means, ranging from in situ buoys, fleet of ships to remote sensing by satellite and aircraft, could be considered, emphasising that each means and any combinations among may act simultaneously. This study focuses on just one of the means; the use of ships as sailing wave buoys. The article introduces the wave buoy analogy, i.e. shipas-a-wave-buoy, and it makes a proposal on how to impose (different) weights to the single ship-specific wave spectrum estimates obtained from multiple ships. Moreover, the work includes a discussion about the importance to associate a measure to reflect the (un)certainty of the wave spectrum estimate. The article presents a numerical case study, where multiple ships act simultaneously as wave spectrum-estimators. The case study relies on numerical motion simulations, as appropriate full-scale data is not yet available. In the analysis, it is shown that the use of simultaneous data from multiple ships leads to more accurate wave spectrum estimations.
\end{abstract}

Keywords:

Sea state estimation, wave buoy analogy, multiple ships, RAO-based weighting, uncertainty measure

Email address: udn@mek.dtu.dk (Ulrik D. Nielsen) 


\section{Introduction}

Generally, waves compromise safe and fuel efficient operation of marine vessels. It is therefore essential to possess knowledge about the onsite wave system, or generally the sea state, since predictions and analyses of wave-induced loads and responses otherwise are difficult to make. This relates directly to the development of the (fully) autonomous ships, where a high-level risk-based supervising controller provides set-points to the lowlevel control-execution controller $[1,2]$. Today, these kind of decisions typically rely heavily on the operator's experience, situational awareness and understanding of risk. Thus, it is understood that if the fully autonomous ship should become a reality, realtime operability assessment and ship motion analysis must be made automatically. For instance, any "system-made" decisions about vessel forward speed and heading relative to waves should rely on accurate modelling of the wave-induced responses. This will, however, only be feasible with accurate estimates of the on-site sea state. Even without reference to ship autonomy, it could be said that sea state estimation is fundamental in the strive towards maximized operability and risk management of ships and ocean structures.

Typical means for sea state estimation (SSE) include classical wave buoys, remote sensing by aircraft and satellite, marine wave radar measurements, and ship-as-a-wavebuoy; all of which are usually applied on an individual basis and herein referred to as observation platforms. In the general context of marine operations (navigating vessels, crane operations, maintenance of offshore windfarms, etc.) there is no one method for SSE that is clearly best, as the individual means all have their own pros and cons, depending on the context [3-7]. For example, the classical wave buoy is probably the most reliable means if concern is about obtaining accurate estimates and wave statistics at a specific geographic location; however, wave buoy data may be of little value if the sea state at a ship's ocean route needs to be monitored.

The main idea presented in this paper is to fuse together measurements from different observation platforms in order to provide improved SSE on both a local on-site position, as well as on a more global geographic scale, also enabling forecasting of a future sea state to be expected ahead of time at a given geographical site.

In this initial study, fusing the wave estimates obtained from multiple ships is con- 
sidered. The particular approach of using the ship as a wave buoy (wave buoy analogy) is not new [8-22], but the idea to consider multiple ships simultaneously has not been studied before. It is noteworthy that the study/approach considers the vessels to be sufficiently spaced, so that ship-to-ship interactions will be negligible. However, at the same time, the ships are not more separated than the statistical properties of the surface wave elevation are the same at all ships' position. That is, any change in the distribution of wave energy density, over space and time, is neglected.

The remaining part of the article consists of five sections. The main principle of the wave buoy analogy, and the reason to have particular focus on (multiple) ships as sailing wave buoys are briefly presented in the following section. The wave buoy analogy can be based on various mathematical procedures, and Section 3 presents one of the methods that can be applied to process wave-induced motion data from a ship. With data from multiple ships it becomes natural to introduce a weighting of the individual ship-specific estimates for what reason Section 4 makes a proposal on how to possibly do this. Section 5 includes a numerical case study used to illustrate the potential improvements that can be expected, when multiple ships are applied simultaneously as sailing wave buoys. Finally, concluding remarks are given in Section 6 .

\section{Multiple ships as sailing wave buoys}

Most of today's marine vessels are instrumented with sensors to record, for instance, wave-induced acceleration levels, the rolling angle, hull girder stresses amidships, etc., see Figure 1. In this sense, ships resemble classical wave buoys, and the response recordings from ships can be processed to facilitate estimation of the on-site sea state [23]; making the analogy to wave buoys by relating the measurements and the sea state through a mathematical model. However, in general, three aspects make the estimation problem more complicated: (1) Ships have more complex geometrical forms compared to wave buoys, for what reason theoretical calculations of the ship motion dynamics in terms of the wave-to-motion transfer functions (RAOs) may have larger uncertainty associated than corresponding calculations for wave buoys. Uncertainties in RAOs may also be imposed because of uncertain operational conditions. (2) Ships generally advance relative to the progressing waves. Consequently, a ship encounters the waves at a different period 


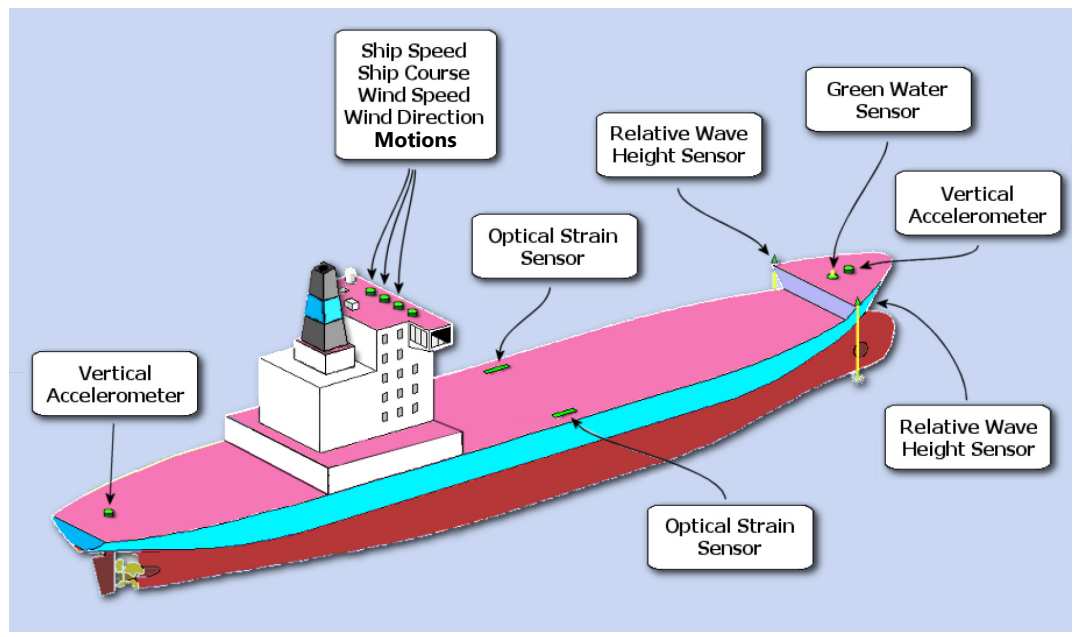

Figure 1: Illustration of (conceptual) sensor arrangement on a vessel [27].

(equivalently, frequency) than does an observer being fixed relative to the inertial frame of reference (i.e., the 'absolute domain'). Physically, this phenomenon is described by the Doppler Shift [24-26] that must be strictly introduced, as theoretical calculations of the wave-induced responses of the ship otherwise cannot be compared with corresponding measurements that are obtained in the 'encounter-domain'. The mathematical solution is to directly establish the governing equations in absolute domain, e.g. [8, 9, 13, 20], or to solve the problem in encounter-domain and, subsequently, convert the solution to absolute domain $[12,25]$. (3) A ship act as a low-pass filter, making the ship less responsive to high-frequency waves. The filtering characteristic depends on ship size (length, breadth, draught) relative to wave length; and various studies consider this particular problem $[21,28,29]$.

The use of ships as sailing wave buoys is believed to be key for a successful development of a networked-based approach for sea state estimation; simply due to the fact that a lot of ships travel most parts of the world's oceans ("quantity is a quality of its own"), see Figure 2.

\subsection{Communication and sharing of data}

At sea, data and information may generally be communicated by Marine Broadband Radio (MBR) and other mobile units via satellites. In this sense, communication channels 


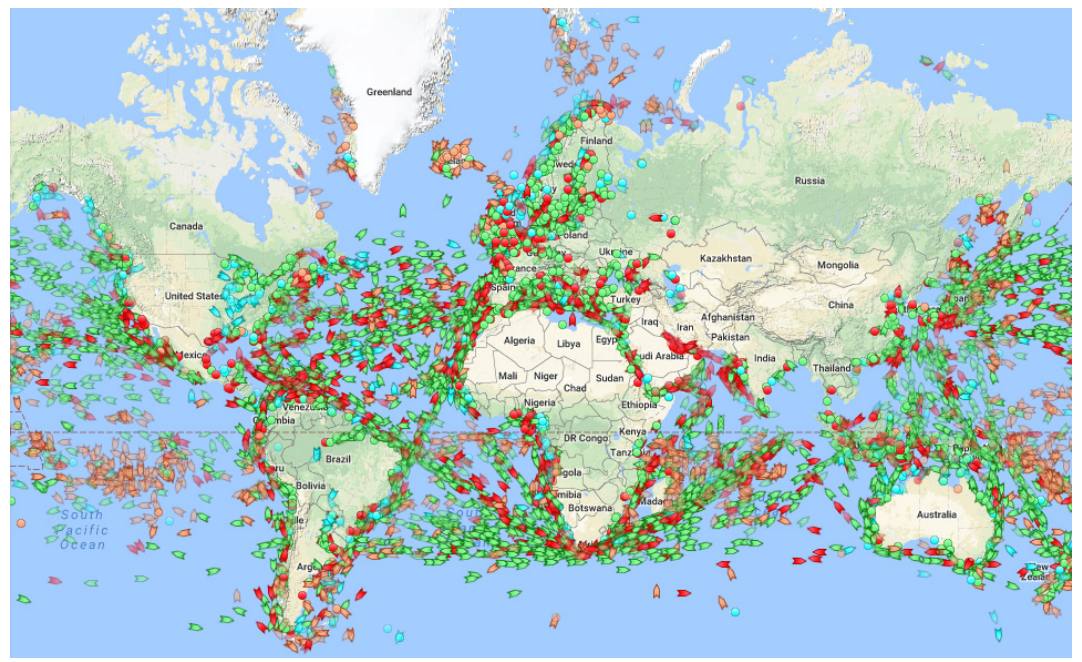

Figure 2: A snapshot of geographic vessel positions based on data from AIS (Automatic Identification System). With permission by MarineTraffic (www.marinetraffic.com/).

for data sharing among multiple ships are already in place. On the other hand, it is not given which data to be sharing. Presently, transmission speed is restricting the sharing - in real-time - of entire data sets; for example, it is not feasible to live-share the full set of wave-induced response recordings from an in-service operating vessel. Thus, it is matter of deciding which kind of data to be sharing. As a likely scenario, real-time data sharing may initially be focused on just characteristic wave parameters such as significant wave height, wave period, and wave direction. However, any further discussion about data communication and sharing is beyond this paper's scope. In this article, it will be assumed that all necessary data, including communication and sharing, is momentarily available on ad-hoc basis, as needed.

As a related note, communication and sharing of data from navigating ships are already in study in the Automatic Identification System (AIS), and a comprehensive survey in this regard has been given by [30]. AIS data is, indeed, a reason why navigating ships are foreseen to have a vital role in the present context (cf. Fig. 2).

\subsection{Assumptions about the sea state and vessel RAOs}


Generally, as any ocean wave system progresses over (larger) distance on open water, various physical phenomena may lead to changes in the wave system's energy distribution (with frequency and direction); for instance, because of dissipation due to fluid viscosity and "opposing" winds, and because of dispersion as a result of varying bathymetry. It could be relevant to consider these effects, when dissociated ships are used simultaneously for sea state estimation. However, in the present study, these effects are not considered. The neglect of dispersion and dissipation, respectively, is assumed to be reasonable if deep-water conditions are considered and if the ocean area, in which the sea state is to be estimated, is not too large. In other words, this study assumes the ocean wave system, and hence the underlying wave energy density spectrum, as constant in both space and time.

As already indicated, accurate RAOs are crucial to ensure reliable sea state estimates when a ship is used as a wave buoy (see also the following section). This study assumes that the RAOs of all individual ships are perfect, and, with an additional assumption taking the vessels' loading conditions and other operational parameters to be exactly known, the main reason to not obtain a perfect estimate of the sea state is due to a ship's behaviour as a low-pass filter. This condition, or "problem", is, in fact, the central topic of Section 4, and at present it suffices to note that any uncertainty in the RAOs is neglected.

\section{Ship motion-based wave spectrum estimation using a spectral-residual cal- culation}

In the past, several successful studies about the wave buoy analogy have been conducted, and it is known [23] that different procedures can be applied. Nonetheless, the underlying computational/mathematical methods have been found to suffer in some cases of being too slow or inefficient (although being fairly accurate). Recently, however, an efficient and reliable procedure has been developed [11, 12], where the former study considers station-kept, dynamically positioned ships exclusively, while the latter [12] focuses on ships with a non-zero forward speed, and to some extent represents a generalisation of the former. The case study, Section 5 , introduces wave spectrum estimation based on the work by [12], and the following contains a brief summary of the theoretical formulation.

It is a general characteristic of the wave buoy analogy that it relates the measured 
signals of wave-induced responses with the exciting unknown wave spectrum through a mathematical model that couples the two parts using theoretically calculated transfer functions. Thus, in a short-crested, stationary seaway, the governing equation system reads

$$
R_{i j}\left(\omega_{e}\right)=\int H_{i}\left(\omega_{e}, \mu+\beta\right) \overline{H_{j}\left(\omega_{e}, \mu+\beta\right)} E\left(\omega_{e}, \mu\right) d \mu
$$

for a set $(i, j)$ of responses taken among, say, the heave $(z)$, roll $(\phi)$, and pitch $(\theta)$ motions, i.e. $i, j=\{z, \phi, \theta\}$. The complex-valued transfer functions, $H_{i}\left(\omega_{e}, \mu+\beta\right)$ and $H_{j}\left(\omega_{e}, \mu+\beta\right)$, yield the theoretical relationship between the i-th and the $\mathrm{j}$-th components of the response spectra $R_{i j}\left(\omega_{e}\right)$ and the directional wave spectrum $E\left(\omega_{e}, \mu\right)$ for wave heading $(\mu+\beta)$ where $\mu$ is the direction of the single waves relative to mean wave heading $\beta$. The bar indicates complex conjugate, and the encounter frequency is $\omega_{e}$. It should be understood that the response spectra $R_{i, j}\left(\omega_{e}\right)$ of Eq. (1) is estimated by the measured data while the right-hand side is obtained through the theoretical calculations. The mean (absolute) wave direction $\vartheta$ is derived from the mean wave heading $\beta$ with account for the compass-course of the vessel. The encounter frequency is related to the absolute frequency $\omega_{0}$ through the Doppler Shift,

$$
\omega_{e}=\omega_{0}-\omega_{0}^{2} \psi, \psi=\frac{U}{g} \cos (\mu+\beta)
$$

where $g$ is the acceleration of gravity, and $U$ is the vessel forward-speed. The Doppler Shift imposes an elementary physical problem but, in practice, this shift implies that the wave estimation problem is rather "delicate" to solve for ships with advance speed; making note that, in the end, the absolute wave energy spectrum rather than the encountered wave energy spectrum must be estimated. Several textbooks introduce the complications involved because of the Doppler Shift, e.g. [31-33], but leaving no solution on how to deal with the problem in practice, when a wave spectrum shall be transformed from encounter frequency-domain to absolute frequency-domain; a process that theoretically cannot be uniquely solved, as it relates one single encounter frequency to three absolute frequencies in following seas. On the other hand, interrelated studies $[25,26]$ outline explicitly an elaborate and practical solution strategy for this problem.

The main (mathematical) task of the wave estimation problem is to solve Eq. (1) for the unknown wave spectrum. The actual solution follows from an iterative approach, 
schematically written as;

$$
\begin{aligned}
& \hat{R}_{i j}\left(\omega_{e}\right)=\hat{S}_{i j}\left(\omega_{e}\right) \int H_{i}\left(\omega_{e}, \mu+\beta\right) \overline{H_{j}\left(\omega_{e}, \mu+\beta\right)} \varphi(\mu) d \mu \\
& \tilde{R}_{i j}\left(\omega_{e}\right)=R_{i j}\left(\omega_{e}\right)-\hat{R}_{i j}\left(\omega_{e}\right) \\
& \hat{S}_{i j}^{+}\left(\omega_{e}\right)=\hat{S}_{i j}\left(\omega_{e}\right)+h_{i j} \tilde{R}_{i j}\left(\omega_{e}\right)
\end{aligned}
$$

performed for any pair $(i, j)$ of response signals for the entire set of considered encounter frequencies. The directional wave spectrum $E\left(\omega_{e}, \mu\right)$ is taken as the product between a point wave spectrum $S_{i j}\left(\omega_{e}\right)$ and a spreading function $\varphi(\mu)$, e.g. [31]. The iteration is initiated by setting the estimate of the wave spectrum $\hat{S}_{i j}\left(\omega_{e}\right)$ equal to zero; emphasising that $\hat{S}_{i j}\left(\omega_{e}\right)$ is computed for each combination of signals. As seen, the iteration builds on a residual calculation (Eq. $3 \mathrm{~b}$ ), where $\hat{R}_{i j}\left(\omega_{e}\right)$ is subtracted from the measured response spectrum $R_{i j}\left(\omega_{e}\right)$ and, hence, forming the residual $\tilde{R}_{i j}\left(\omega_{e}\right)$. Subsequently, the residual facilitates an update (Eq. 3c) of the wave spectrum estimate, $\hat{S}_{i j}^{+}\left(\omega_{e}\right)$, using a prescribed gain $h_{i j}>0$. This iteration is continued until a threshold is reached for the residual $\left|\tilde{R}_{i j}\right| \leq \epsilon$, for $\epsilon>0$.

The iteration scheme (Eqs. 3a-3c) is as mentioned applied for each signal-combination $(i, j)$, and in addition the iteration is performed for a set of discrete wave headings on the half circle (0-180 deg.), for port/starboard symmetric ships, since the wave direction is not known a priori. Whether the waves are approaching from port or starboard side is determined after the iteration has terminated. Altogether, the solutions obtained from Eqs. (3a-3c) form a set of initial estimates of the encounter-wave energy spectrum. Thus, in order to obtain the final wave spectrum estimate $S\left(\omega_{0}\right)$, valid in absolute domain, the initial brute-force solutions, $\hat{S}_{i j}\left(\omega_{e}\right)$, must be post-processed. The reader is referred to [12] for details.

In a following section, a case study is considered to illustrate how sea state estimation can be done - and improved - by using multiple ships as sailing wave buoys. Before this, however, it is essential to discuss how to introduce weight factors, based on the waveto-motion transfer functions of the individual ships, and, furthermore, how to associate (un)certainty to the actual wave spectrum estimate. 


\section{Weighting of the ship-specific estimates and (un)certainty measure}

This section discusses two relevant aspects of wave spectrum estimation using multiple ships. (i) Weighting of the estimate from each ship based on the wave-to-motion transfer functions of the specific ship. This includes a weighted estimate of the wave direction. See subsection 4.2. (ii) Associating an uncertainty measure to the weight-averaged wave spectrum estimate. See subsection 4.3.

\subsection{Conditions}

In the following discussions, the conditions and assumptions are:

- Totally, $K$ ships are considered, and index $k=\{1,2, \ldots, K\}$ is used to characterise a specific ship. Hence, there will be $K$ wave spectrum estimates, $\hat{S}_{k}\left(\omega_{0}\right), k=$ $1,2, \ldots, K$, that can be used collectively to derive one (final) weight-averaged wave spectrum from. The weight-averaged spectrum is denoted $\hat{S}^{0}\left(\omega_{0}\right)$.

- The ship-specific wave spectrum estimate $\hat{S}_{k}\left(\omega_{0}\right)$ obtained from the single ship is derived by the wave buoy analogy using the 'brute-force spectral' approach described in Section 3, which is a summary of [12].

- Weighting of the individual wave spectrum estimates is made solely on the basis of the single sets of transfer functions of the group of ships considered. Other "weighting metrics", for instance based on the relative distances between the individual vessels, are not included.

- For any ship, the considered set of motion components are heave, roll, and pitch, i.e. $i=\{z, \phi, \theta\}$, cf. Section 3. Reference to a given ship's set of transfer functions is according to $H_{i, k}(\ldots)$.

- Angular $[\mathrm{rad} / \mathrm{s}]$ and time-wise frequency $[\mathrm{Hz}]$ are used interchangeably but it is absolute frequency being considered no matter the choice. The angular frequency $\omega$ and time-wise frequency $f$ are related by $\omega=2 \pi f$.

- The physical distances between the ships are at all times assumed to be no more than what allows for statistically identical sea states, including wave direction, at 
the vessels' positions. At the same time, the vessels are so far apart that interactions between the vessel hulls do not occur.

\subsection{Weighting of the single wave spectrum estimates}

The central point in the following is to establish a set of frequency-dependent and ship-specific weight functions. The individual weight functions should be multiplied with the wave spectrum estimate from the single ships to give the particular estimate greater or smaller (frequency-dependent) trust. The level of trust in a frequency region is dependent on the particular vessel's filtering characteristic, where any of the six degrees-of-freedom (DOF) motion components, in principle, can be utilised. In the following, the heave and pitch filtering characteristics are considered.

The behaviour of a ship as a (linear) low-pass filter means that the given ship "filters away" high-frequency wave components in its motion response. The filtering characteristics are described by the (wave-to-motion) transfer functions, and are generally dependent on the vessel's size and shape. Consequently, it makes sense to introduce a weighting of the single ship-specific wave spectrum estimate based on the given ship's transfer functions. Note, the transfer function $H_{i, k}(\ldots)$ is complex-valued or, equivalently, represented by a corresponding pair of modulus (i.e. magnitude) and phase, see also Section 3 . In the following, only modulus $\left|H_{i, k}(\ldots)\right|$ is considered although this is not necessarily stated.

The sets of transfer functions $\left|H_{i, k}(f \mid \beta)\right|$ of heave and pitch for three different vessels* are shown in Figure 3. In the figure, the individual subplots show the modulus at different encounter angles $\beta$; where the thinner (coloured) lines represent specific encounter angles between 0 deg. (following sea) and $180 \mathrm{deg}$. (head sea). In the further contemplations, the actual variation with encounter angle is not considered, since the 'mean curve', obtained as the average of all single curves for a given frequency, is used,

$$
\bar{H}_{i}(f)=\operatorname{mean}_{\text {All } \beta}\left[\left|H_{i, k}(f \mid \beta)\right|\right]
$$

This curve is shown as the bold, blue line in the individual sub-plots of Figure 3.

The weighting functions are based on normalisations of Eq. (4), since vessels of different size generally may have (very) different transfer function magnitudes, depending

\footnotetext{
${ }^{*}$ The vessels are exactly the same as those used later in the case study in Section 5 .
} 
on the considered motion component, as seen for the modulus of pitch from the three right-hand side plots in Figure 3. The normalised modulus of any motion component is denoted by $\alpha_{i}(f)$, which applies to a specific frequency $f$, and normalisation is made with respect to the maximum value of the (averaged) modulus,

$$
\alpha_{i, k}(f)=\frac{\bar{H}_{i, k}(f)}{\max \bar{H}_{i, k}(f)}
$$

Figure 4 shows the normalised moduli for heave (left) and pitch (right) for the three respective vessels.

To arrive at one frequency-dependent weight function $\varrho$ applicable to a given motion component and to a given ship, the specific normalised modulus of the particular ship is weighted with consideration to the normalised moduli of all considered vessels. Hence, the weight function is defined by,

$$
\varrho_{i, k}(f)=\frac{\alpha_{i, k}(f)}{\sum_{k} \alpha_{i, k}(f)}, \quad k=1,2, \ldots, K
$$

where it is noted that $\varrho$ by definition takes values between 0 and 1 ; it is 0 if the particular ship, denoted by $k=k_{0}$, has no response at the given frequency $f$, i.e. $\bar{H}_{i, k=k_{0}}(f)=0$, while it is 1 at any frequency if $\bar{H}_{i, k=k_{0}}(f)>0$ and $\bar{H}_{i, k \neq k_{0}}(f) \equiv 0$. The weight functions
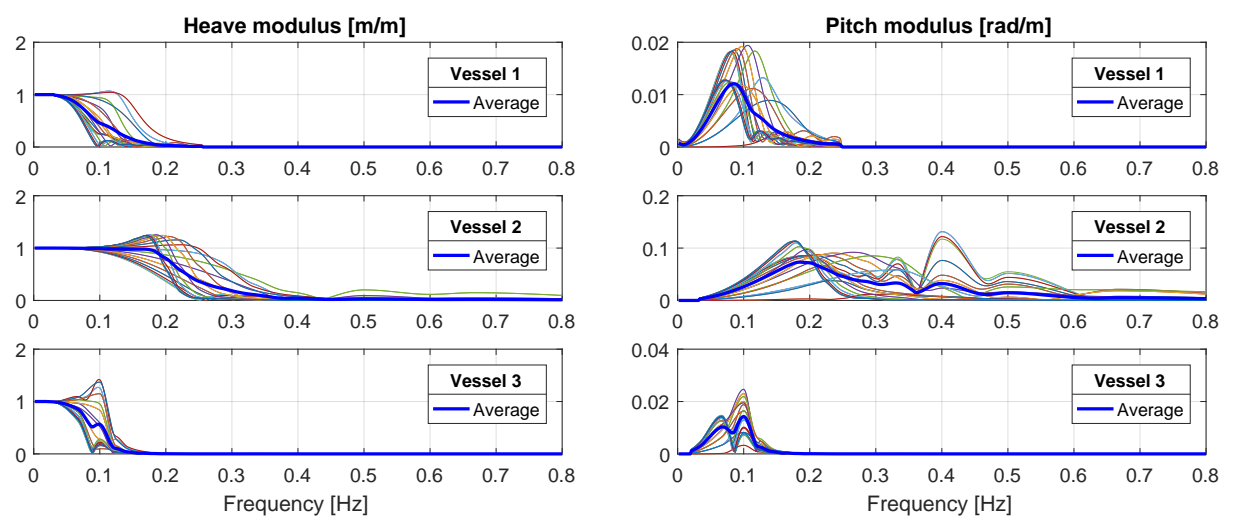

Figure 3: The filtering characteristics in terms of transfer function magnitudes of three sizewise different vessels; heave is the left- and pitch the right-hand side plots, where each plot shows the results for different wave-encounter angles. Note the difference in scales on the y-axes on pitch magnitudes. 
for heave and pitch are shown in Figure 5. For instance, it is seen that the pitch-based weighting factor for vessel 2 takes a value approximately equal $\varrho_{\theta, 2}(0.10)=0.17$ at the frequency $f=0.10 H z$. This value is obtained as $\frac{0.37}{0.85+0.37+1.00} \approx 0.17$ with reference to the plot of the normalised pitch moduli (right-hand side plot in Figure 4).

With the set of weight functions $\varrho_{i, k}(f)$ available, two remarks are noteworthy. Remark 1: As already indicated, the hypothesis is that just one of the weight functions should be used to arrive at a weight-averaged (overall) wave spectrum estimate in a given operational scenario. Which one of the weight functions, the heave- or the pitch-based, to use may be chosen subjectively or by some objective criterion; although it is beyond the scope of the present paper to make a study of such an objective criterion. Herein, it is therefore a matter of selecting the one or the other, but, in any case, the (final)
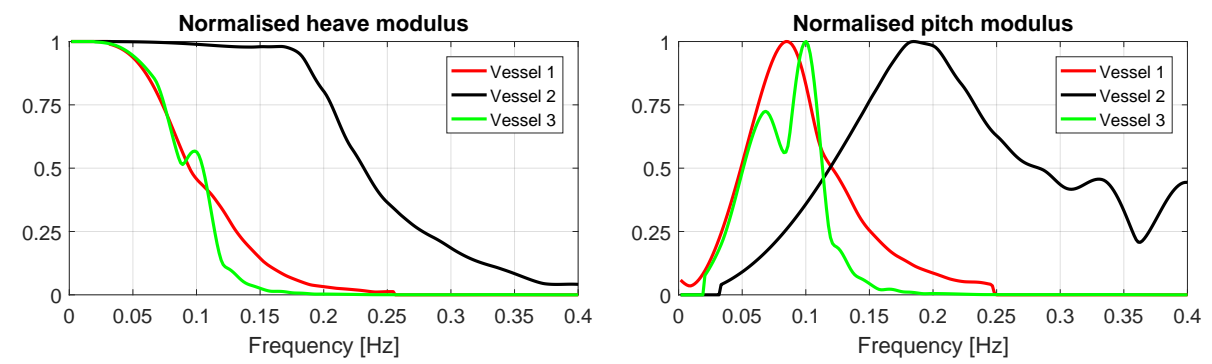

Figure 4: The normalised moduli (of 'mean curves') of heave (left-hand side) and pitch (righthand side). Normalisation is made with respect to the maximum value of the 'mean curves', cf.

Figure 3
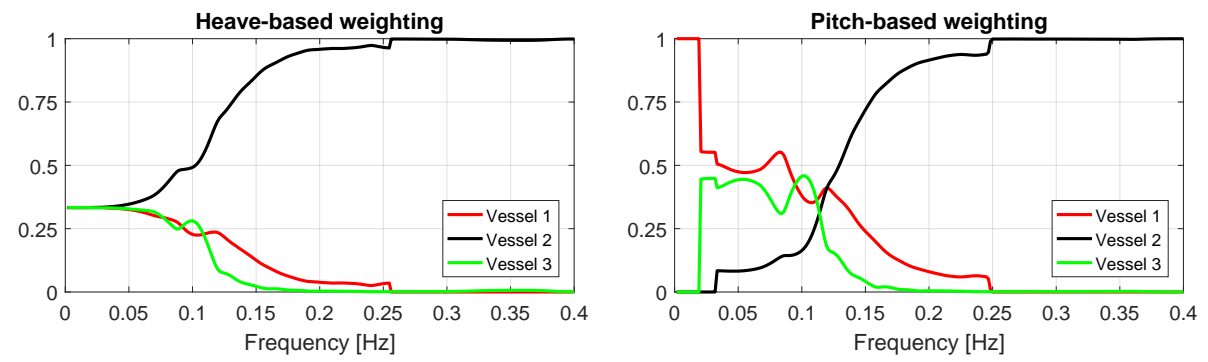

Figure 5: Ship-specific heave and pitch-based weighting functions derived from the moduli of corresponding transfer functions. 
weight-averaged wave spectrum estimate is obtained from,

$$
S_{i}^{0}\left(\omega_{0}\right)=\sum_{k} \varrho_{i, k}\left(\omega_{0}\right) \cdot S_{k}\left(\omega_{0}\right)
$$

emphasising that index $i$ refers to the specific motion component upon which the weighting function is established and index $k$ refers to a given ship. As an example, if the pitch-based weight function $(i=\theta)$ from Figure 5 is used to compute an average wave spectrum estimate in some operational scenario, it will be found that the averaged estimate is primarily based on the estimates from Vessels 1 and 3 at the lower frequency region [0.02-0.10] Hz. On the contrary, for frequencies larger than about $0.20 \mathrm{~Hz}$, the averaged wave spectrum estimate will be (almost) entirely based on the wave spectrum estimate from Vessel 2; in the intermediate region [0.10-0.15] $\mathrm{Hz}$, the wave spectrum estimates from all three vessels are weighted somewhat equally. Note, with little need to mention, it should be realised that the sum of weight factors at any given frequency always add to 1, cf. Eq. (6), independently on the considered motion component. Remark 2: The weight function of a specific ship is based on the normalised modulus obtained from the mean transfer function magnitudes over all directions. The reason to not take relative wave direction into account in the weighting function is that the true relative wave direction, in principle, is not known. In practice, however, the relative wave direction is estimated by each ship, but using this as input to the weight function would make the uncertain estimate influence the weight functions.

\subsubsection{Estimation of wave heading.}

As noted, the single ship-specific wave spectrum estimate is associated with an estimate $\hat{\beta}_{k}$ of the mean wave heading. Equivalently, the mean wave direction $\hat{\vartheta}_{k}$ can be obtained if $\hat{\beta}_{k}$ is combined with vessel no. $k$ 's compass course. As multiple ships are considered simultaneously, it makes sense to introduce a weighting of the individual wave direction estimates $\hat{\vartheta}_{k}, k=1,2, \ldots, K$; for instance, using a similar filtering-based approach like the one introduced for the wave spectrum, cf. Eq. (7). For the wave direction, however, the weighting is not frequency dependent but, instead, it is suggested to calculate ship-specific weight factors $\Lambda_{i, k}$ to be multiplied with the single wave direction 
estimates associated with the given ships. Herein, the weight factors are defined by,

$$
\begin{aligned}
\lambda_{i, k} & =\int_{0}^{\infty}\left|\varrho_{i, k}\left(\omega_{0}\right)\right|^{2} S^{0}\left(\omega_{0}\right) d \omega_{0} \\
\Lambda_{i, k} & =\frac{\lambda_{i, k}}{\sum_{k} \lambda_{i, k}}
\end{aligned}
$$

repeating that indices $i$ and $k$ refer to a particular motion component and a specific ship, respectively. It is noted that $\lambda_{i, k}$ resembles the 0th order spectral moment; but emphasising that the previously defined weight functions (Eq. 6) are used rather than the moduli of the actual transfer functions. The weight-averaged wave direction estimate is

$$
\vartheta_{i}^{0}=\sum_{k} \Lambda_{i, k} \cdot \vartheta_{k}
$$

The index $i$ on the wave direction estimate $\vartheta_{i}^{0}$ indicates that some sort of criterion should/could be introduced to select the motion component upon which the weight factor $\Lambda_{i, k}$ should be derived from; see also Remark 1 above. In the case study below, both heave and pitch are used to establish ship-specific weight functions $\varrho_{i, k}\left(\omega_{0}\right)$ and factors $\Lambda_{i, k}$ from.

\section{3. (Un)certainty measure of wave spectrum estimate}

Qualitatively, the frequency-wise variation, or deviation, between the individual shipspecific (un-weighted) wave spectrum estimates $S_{k}\left(\omega_{0}\right)$ reflects a level of (un)certainty. Thus, as a hypothesis, it is assumed that (almost) equal spectral density estimates at any frequency indicate a high level of certainty at the given frequency, while larger deviations between the spectral density estimates reflect larger uncertainty at the given frequency. It must be stressed that the calculation of deviations between the ship-specific estimates has to be made before any sort of weighting is introduced. A quantification of the preceding is obtained by the frequency-dependent measure $\Delta \equiv \Delta\left(\omega_{0}\right)$ defined by,

$$
\left|\Delta\left(\omega_{0}\right)\right|^{2}=\sum_{k=1}^{K-1} \sum_{l=k+1}^{K}\left[\left|S_{k}\left(\omega_{0}\right)-S_{l}\left(\omega_{0}\right)\right|^{2}\right]
$$

repeating that index $k$ refers to a particular ship, and noting that $\Delta\left(\omega_{0}\right)$ should be calculated for a discrete set of frequencies. It is noted that for $K=3$, Eq. (11) reads 
$\left|S_{1}\left(\omega_{0}\right)-S_{2}\left(\omega_{0}\right)\right|^{2}+\left|S_{1}\left(\omega_{0}\right)-S_{3}\left(\omega_{0}\right)\right|^{2}+\left|S_{2}\left(\omega_{0}\right)-S_{3}\left(\omega_{0}\right)\right|^{2}$. Moreover, the measure resembles basically that of a frequency-wise 'standard deviation'.

Now, the matter of subject is to calculate the "accumulated", or total, (un)certainty $\psi$ by considering the whole range of (discrete) frequencies. This is achieved by making the frequency-integrated version of Eq. (11), and relate it to the energy level of the (estimated) sea state. The energy level of a sea state is generally represented by the 0 -th order spectral moment, obtained as the area under the wave spectrum that characterises the sea state. In this case, the characterising sea state is taken as the weight-averaged (final) wave spectrum estimate $S^{0}\left(\omega_{0}\right)$, see Subsection 4.2 and Eq. (7). Hence, the (un)certainty measure is calculated as

$$
\psi=\frac{\frac{1}{K} \int_{0}^{\infty} \Delta\left(\omega_{0}\right) d \omega_{0}}{\int_{0}^{\infty} S^{0}\left(\omega_{0}\right) d \omega_{0}}
$$

accounting for $K$ ships in total. By definition, $\psi=0$ represents perfect agreement between all of the $K$ ship-specific wave spectrum estimates considered; that is, the spectra are truly identical. On the other hand, the larger $\psi$ is, the larger is the deviation between the single estimates, which in turn represents increasing degree of uncertainty. The (un)certainty measure $\psi$ is not an absolute measure but should instead be considered relatively. It does not indicate if a wave spectrum estimate is good (or bad), equivalently how close the estimate is to the $\operatorname{truth}^{\dagger}$. Per definition, $\psi$ is simply a measure that expresses how much the single ship-specific estimates deviate from each other. Thus, $\psi=0$ does not necessarily indicate a perfect estimate, as it could be the result of an extreme case where all ship-specific estimates were identical, but otherwise off the truth. However, the measure of $\psi$ should - with reasonable assumptions and as-good-as-possible data - be a measure that can be used to infer about the likeliness that a particular wave spectrum estimate is good in given operational conditions.

It is noteworthy that the proposed (un)certainty measure realises by fusing together multiple estimates of the same sea state. In addition, it would be relevant to propose a measure to be associated with the wave spectrum estimate from just a single ship. Work

\footnotetext{
${ }^{\dagger}$ Note, the exact truth itself is never known although estimates from other means (buoys, satellites, ...) may be available.
} 
in this direction has been initiated by [34] that introduces uncertainty modelling of the RAOs. In the present study, however, uncertainty is considered/computed entirely by the calculation of $\psi$ in Eq. (12), since any uncertainty associated with the ships' RAOs is neglected, cf. Section 2.

\section{Case study: Multiple ships as sailing wave buoys}

This section presents a case study based on numerically simulated data. The case study builds on an operational scenario where three ships are used simultaneously as sailing wave buoys, see Section 3. The actual ships are selected somewhat arbitrarily albeit they are chosen in such a way that they reflect different filtering characteristics to better illustrate the discussions in Section 4 . The ships will be presented in the following, but their pertinent data has already been shown in Figure 3 presenting the moduli of the sets of transfer functions.

The spectral residual approach, Section 3, can be complemented with a stability analysis [35] to find suitable values for the iteration gains $h_{i j, k}$, cf. Eq. (3b) to improve performance. However, this exercise is beyond the scope of the present study. It is noteworthy, too, that there are other mathematical models of the wave buoy analogy available; an account has been given by [23]. The prime argument to use the spectralresidual approach, cf. Section 3, in favour of other available models, is the fact that it has high(er) computational efficiency, and, at the same time, yields reasonable results $[11,12,35,36]$.

\subsection{Simulated motion measurements of three ships}

Wave-induced motion measurements have been simulated for three vessels, all operating in a given confined ocean area. The three vessels are taken as an advancing medium-sized Ro-Ro vessel ('Vessel 1'), an advancing smaller research vessel ('Vessel 2'), and a station-kept FPSO ('Vessel 3'), respectively. The specific vessels are characterised by main dimensions as listed in Table 1. The wave-to-motion transfer functions of the Ro-Ro ship and the research vessel (R/V) have been calculated by linear strip theory, while a linear potential theory-based panel code has been used for the FPSO. The motion transfer functions have been computed for all six degrees of freedom, considering the 
Table 1: Main dimensions of the three vessels, a Ro-Ro ship, a research vessel (R/V), and an FPSO, used as sailing wave buoys.

\begin{tabular}{lcccc}
\hline Vessel & $\begin{array}{c}\text { Length } \\
{[\mathrm{m}]}\end{array}$ & $\begin{array}{c}\text { Breadth } \\
{[\mathrm{m}]}\end{array}$ & $\begin{array}{c}\text { Draught } \\
{[\mathrm{m}]}\end{array}$ & $\begin{array}{c}\text { Block coef. } \\
{[-]}\end{array}$ \\
\hline Ro-Ro & 232.0 & 33.0 & 6.1 & 0.61 \\
R/V & 28.9 & 9.6 & 2.7 & 0.56 \\
FPSO & 200.0 & 44.0 & 12.0 & 0.79 \\
\hline
\end{tabular}

whole range of wave-encounter angles (0 - 360 deg.), spaced at 10 deg., and the sets of cut-off frequencies are reflected by the plots of the moduli of the transfer functions, see Figure 3. Note, onwards the three vessels are referred to by their type-specific names, i.e. Ro-Ro, R/V, and FPSO.

The motion responses are simulated using standard procedure considering shortcrested waves and assuming the wave elevation as a Gaussian process, see e.g. [12]. Realisations are made for the motion components of heave, roll, and pitch, respectively, for all three vessels.

The operating scenario is illustrated in Figure 6, where it is important to note that the indicated wave system approaches from the same mean direction and is represented by a stationary, and identical, sea state in the whole operational area; that is, the sea state does not change temporally, during the given measurement period, say, in the order of 30 minutes, and remains the same at all the spatial positions of the vessels in any considered operational scenario. The combination of sea state and operational parameters (vessel speeds and course/heading) of multiple ships leads to a large number of possible operational scenarios. This study has its focus on just a few ones to introduce and illustrate the use of multiple ships simultaneously as sailing wave buoys. The results to be presented apply to eight sub-scenarios in total, distinguished by different sea states and/or different operational parameters. Thus, four main scenarios are defined according to sea state variations, A, B, C, and D, while for each wave scenario two combinations of the operational parameters are considered, 1 and 2. The sub-scenarios are presented in Table 2. It is seen that sea states A and B represent wave conditions described by a unimodal wave spectrum, here taken as a Bretschneider spectrum overlaid with a spreading function to introduce short-crested waves, e.g. [31]. Sea states C and D 
characterise wave conditions observed during the existence of wind waves and swell at the same time, and approaching from the same direction. Specifically, the respective sea states $\mathrm{C}$ and $\mathrm{D}$ are defined by the summation of two Bretschneider spectra, again overlaid with a spreading function to resemble directional, short-crested waves. The theoretically known 'generating wave conditions' are used to simulate sets of motion recordings of the ships for given (true) wave headings $\beta_{1}, \beta_{2}$, and $\beta_{3}$, and vessel speeds, $U_{1}, U_{2}$, and $U_{3}$, respectively. The wave heading, equivalently the encounter angle, $\beta$ is defined as the angle between the mean direction $\vartheta$ of the propagating waves and the individual ship's centerline, and $\beta=180 \mathrm{deg}$. is head sea.

Based on the specified sea state and a set of operational parameters, cf. Table 2, motion time history recordings of \{heave, roll, pitch\} have been simulated for each vessel for 30 minutes. Consequently, the simulated "measurements" represent one single 30-minutes realisation out of infinitely many comprising the whole ensemble. Strictly

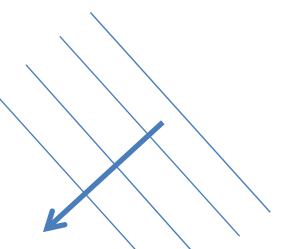

Waves
Vessel 2

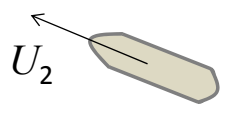

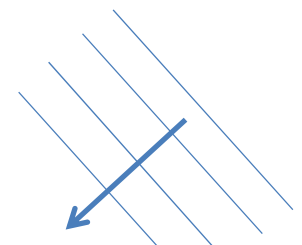

Waves

Vessel 1

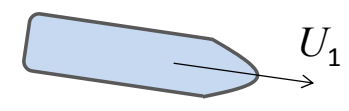

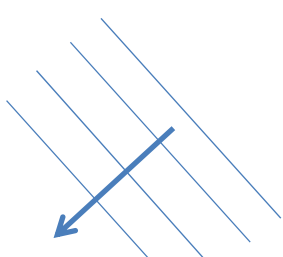

Waves

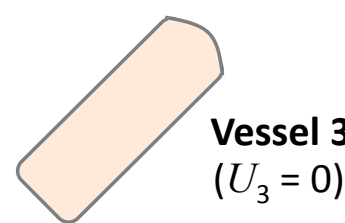

$\left(U_{3}=0\right)$

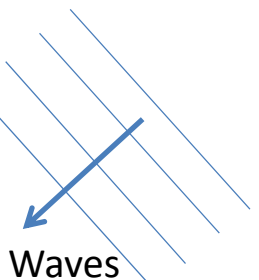

Figure 6: Illustration of the operating scenario where three vessels are used simultaneously for sea state estimation. The sea state and the mean wave direction are the same in the whole operational domain and, likewise, it is assumed that the sea state and wave direction do not change in the considered period of time, in the order of half an hour. 
Table 2: Operational conditions in the sub-scenarios and generating sea state at the vessels' point of operation. The (true) sea state is assumed to be the same over the whole ocean area in which the three vessels operate.

\begin{tabular}{|c|c|c|c|c|c|c|c|c|c|c|}
\hline & \multicolumn{3}{|c|}{ Speed [kts] } & \multicolumn{3}{|c|}{ Heading [deg.] } & \multicolumn{2}{|c|}{ Swell } & \multicolumn{2}{|c|}{ Wind sea } \\
\hline & $U_{1}$ & $U_{2}$ & $U_{3}$ & $\beta_{1}$ & $\beta_{2}$ & $\beta_{3}$ & $H_{s}[\mathrm{~m}]$ & $T_{p}[\mathrm{~s}]$ & $H_{s}[\mathrm{~m}]$ & $T_{p}[\mathrm{~s}]$ \\
\hline $\mathrm{A} 1$ & 11 & 10 & 0 & 240 & 030 & 180 & - & - & 3.0 & 8.0 \\
\hline $\mathrm{A} 2$ & 16 & 10 & 0 & 240 & 070 & 180 & - & - & 3.0 & 8.0 \\
\hline B1 & 11 & 10 & 0 & 240 & 030 & 180 & - & - & 3.0 & 12.0 \\
\hline B2 & 16 & 10 & 0 & 240 & 070 & 180 & - & - & 3.0 & 12.0 \\
\hline $\mathrm{C} 1$ & 11 & 10 & 0 & 240 & 030 & 180 & 3.0 & 15.0 & 3.0 & 8.0 \\
\hline $\mathrm{C} 2$ & 16 & 10 & 0 & 240 & 070 & 180 & 3.0 & 15.0 & 3.0 & 8.0 \\
\hline D1 & 11 & 10 & 0 & 240 & 030 & 180 & 3.0 & 17.0 & 3.0 & 10.0 \\
\hline D2 & 16 & 10 & 0 & 240 & 070 & 180 & 3.0 & 17.0 & 3.0 & 10.0 \\
\hline
\end{tabular}

speaking, it is therefore necessary to consider several of these time history realisations in order to properly account for the stochastic nature of ocean waves and, hence, allowing a comprehensive and valid statistical analysis of the 'post-processed outcome', i.e. the estimation of sea states. The following analyses are based on (statistical) evaluations made from sets of 20 time history realisations of \{heave, roll, pitch $\}$ for the Ro-Ro ship, the research vessel $(\mathrm{R} / \mathrm{V})$, and the FPSO, respectively.

\subsection{Results and discussions}

The following bullets summarise a few noteworthy points about the results to be presented:

- Wave spectrum estimation is made using simultaneous data from three ships, i.e. $K=3$. For any given ship, a certain ship-specific estimate is obtained by the brute-force spectral procedure reviewed in Section 3.

- Sets of results are presented for three different weight functions, cf. Eqs. (6) and (8); one set based on an equal weighting of the individual ships, that is, $\varrho_{i, k}\left(\omega_{0}\right) \equiv$ $\Lambda_{i, k}=\frac{1}{3}$, and two other sets based on the heave and the pitch motions, respectively; cf. Figure 5.

- The true ship-specific encounter wave-angle, or wave heading, cf. Table 2, has been subtracted from the single (ship-specific) estimate. The resulting value is "defined" 
as the wave direction $\vartheta_{k}$. Consequently, $\vartheta_{k}$ will be zero for a perfect wave heading estimate; emphasising that this is so for all the considered ships.

- Each operational sub-scenario, cf. Figure 6 and Table 2, is evaluated statistically by the processing and analysis of totally twenty sets of realisations of simulated motion measurements.

\subsubsection{Plots of wave spectra}

Examples of ship-specific wave spectrum estimates are seen in Figure 7, where arbitrarily selected outcomes of realisations from the four sub-scenarios C1-D2 are presented in sub-plots (i)-(iv). In the figure, any sub-plot includes the actual spectrum estimates and the corresponding true generating wave spectrum. A detailed inspection of the

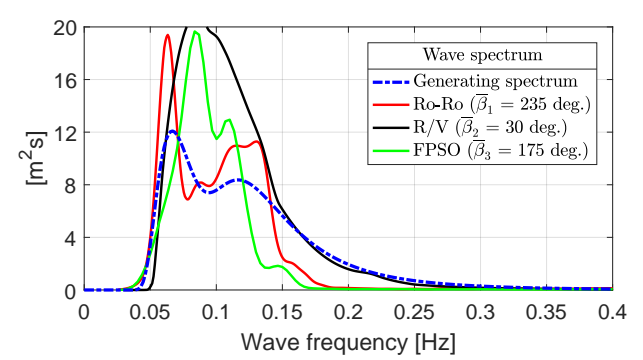

(i) Sub-scenario C1.

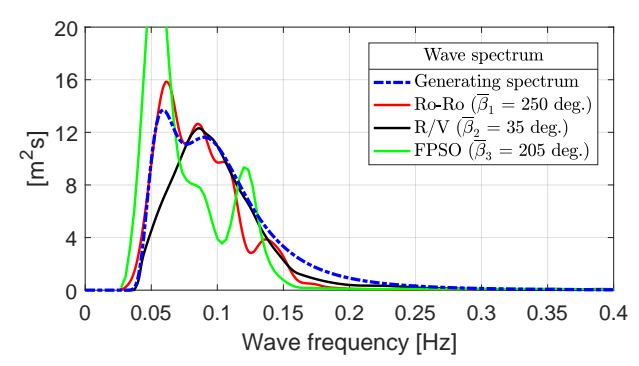

(iii) Sub-scenario D1.

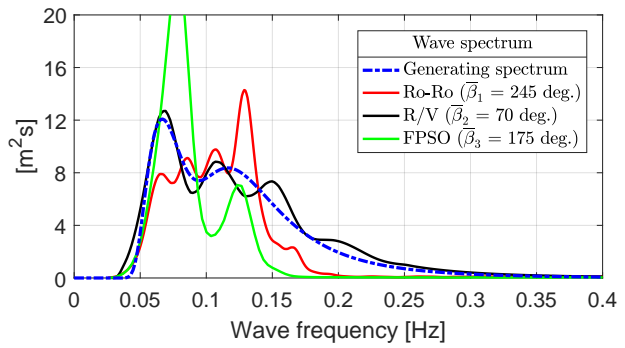

(ii) Sub-scenario C2.

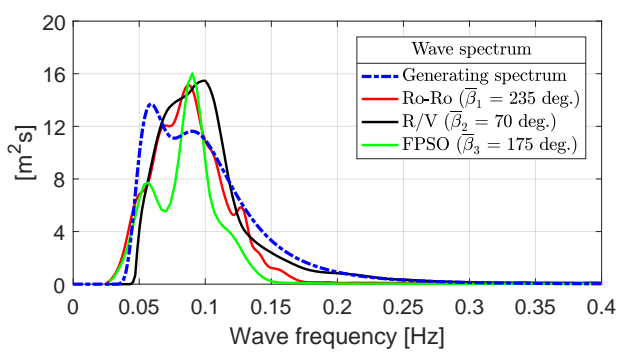

(iv) Sub-scenario D2.

Figure 7: Examples of ship-specific wave spectrum estimates obtained by processing the sets of motion time history recordings from the single vessels. The input-wave spectrum is shown together with the estimates, and the estimated wave heading is included in the subplots' legend. Note, the associated wave heading estimates are included in the plots' legend. 
spectrum estimates in Figure 7 reveals that the two larger vessels, as expected, cannot be used to estimate the higher-frequency components of the wave system; explained because of the vessels' filtering effect. Furthermore, it is seen that the single estimates of all three ships do not match exactly with respect to the number of spectral peaks and the locations of the peaks. However, it is observed that, collectively, the set of spectrum estimates "captures" the spectral density, by and large, on the the whole range of frequencies; a finding applicable to any of the realisations from sub-scenarios $(\mathrm{C} 1, \mathrm{C} 2$, D1, and D2), and also to the remaining four sub-scenarios although not shown. In this sense, it should be interesting to consider all the spectrum estimates simultaneously by introducing a weight-averaged estimate like discussed in Section 4. Thus, the ship-specific wave spectrum estimates have been weighted according to the approach outlined previously, and the outcomes appear from Figure 8. The shown examples correspond to the

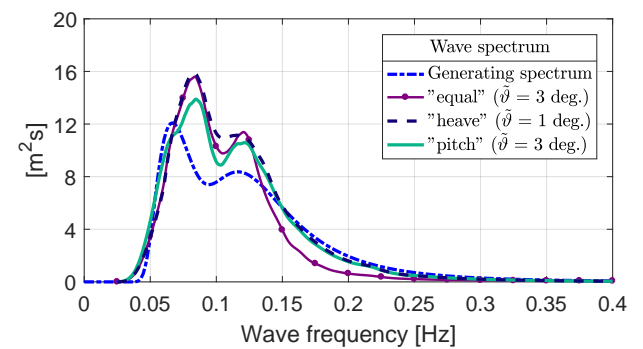

(i) Sub-scenario C1.

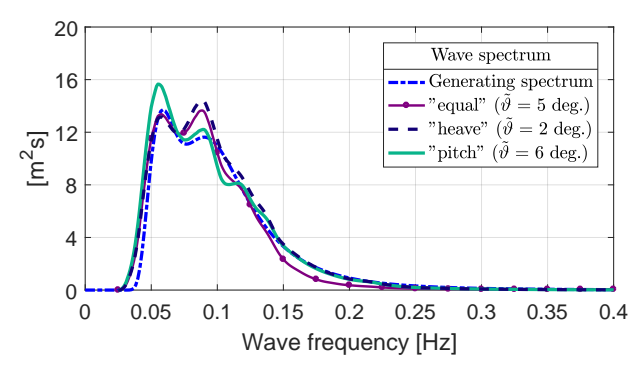

(iii) Sub-scenario D1.

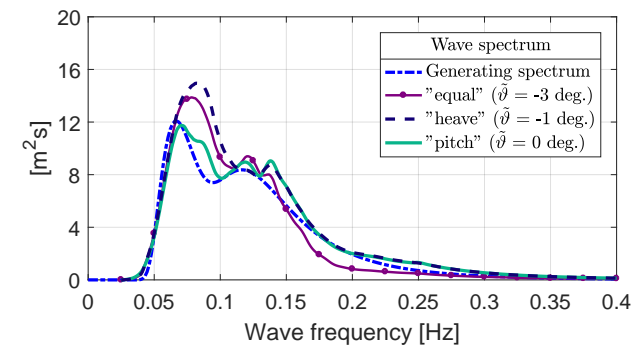

(ii) Sub-scenario C2.

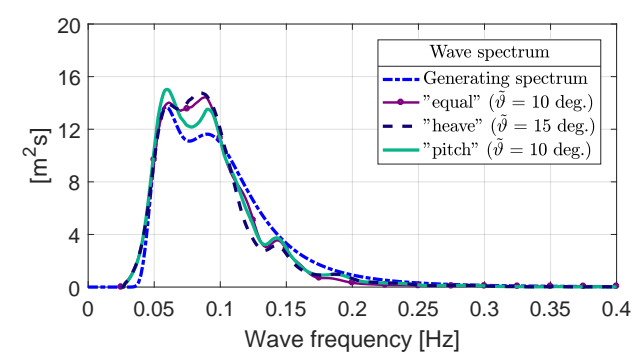

(iv) Sub-scenario D2.

Figure 8: Examples of (corresponding) weight-based mean wave spectrum estimates, cf. Figure 7. Note, the associated wave direction estimates are included in the plots' legend, where $\tilde{\vartheta} \equiv \vartheta^{0}$, cf. Eq. (10); the true value is 0 deg. 
same operational sub-scenarios considered in Figure 7. The sub-plots' legends refer to different weighting functions, where "equal" means that the three individual ship-specific wave spectrum estimates (from Figure 7 ) are weighted equally with $\varrho_{i, k} \equiv \frac{1}{3}$ in Eq. (7). Legends "heave" and "pitch" refer to weighting functions derived from the normalised moduli of the heave and pitch transfer functions; cf. Figure 5. Furthermore, the legends include the weight-averaged wave direction estimate $\vartheta^{0}$ applicable to the particular (weight-averaged) spectrum. It is evident from Figure 8 that all the weighted spectrum estimates ("equal", "heave", "pitch") compare well with the true generating spectrum for all the sub-scenarios; and the agreement is improved considerably compared to the individual ship-specific estimates (cf. Figure 7). It is observed that the two peaks of the wave spectrum are estimated at almost identical frequencies close to the true ones, and also with the spectral values of the peaks in reasonable agreement. Another specific observation from the four sub-plots in Figure 8 is noteworthy; the capability of the weight-averaged estimates to capture the higher-frequency spectral wave components. This is especially true for the heave- and pitch-based results, where the estimate by the (smaller) research vessel is given the most weight at higher frequencies (cf. Figure 5). The mentioned observations apply to all remaining realisations of the four considered operational sub-scenarios and also to the other sub-scenarios (cf. Table 2) and their respective realisations.

In order to evaluate and compare the single weight-averaged estimates and the true generating spectrum, a difference-metric $\varepsilon_{i}$ is used. It is based on the absolute value of the error between the spectral components of the particular weight-averaged estimate $S_{i}^{0}\left(\omega_{0}\right)$, cf. Eq. (7), and the true generating spectrum $S\left(\omega_{0}\right)$,

$$
\varepsilon_{i}=\frac{\int\left|S_{i}^{0}\left(\omega_{0}\right)-S\left(\omega_{0}\right)\right| d \omega_{0}}{\int S\left(\omega_{0}\right) d \omega_{0}}
$$

where index $i$ refers to a weighting-based result derived from the transfer functions of heave or pitch, or from an equal weighting of the ship-specific estimates. Qualitatively, $\varepsilon_{i}$ is the (normalised) "area-deficit", found as that encased by the estimated spectrum and the generating spectrum. Thus, the metric provides, in just a single number, a measure that enables a relative comparison between the different weight-based estimates. 

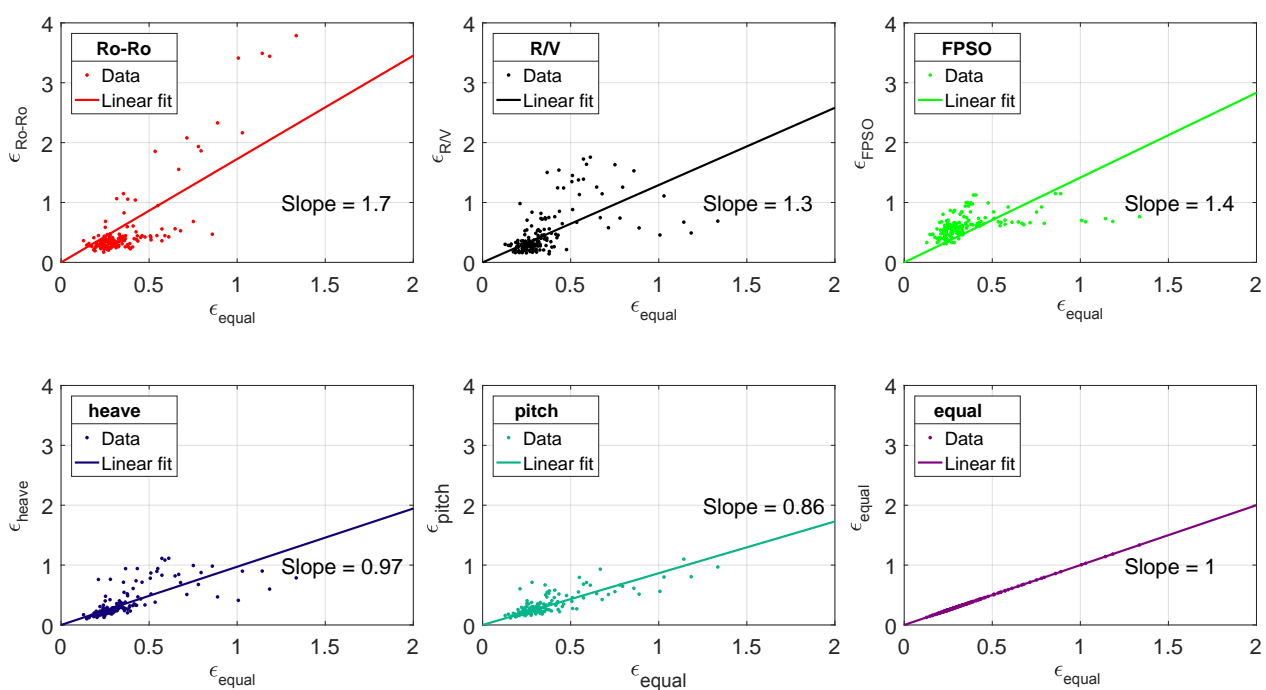

Figure 9: Evaluation of the detailed - but relative - agreement in terms of the error measure $\varepsilon_{i}$ between spectrum estimates and the true generating spectrum for all realisations of all subscenarios. The base-reference is $\varepsilon_{\text {equal }}$. The slope of the fitted linear trendline has been included in the respective sub-plots.

It should be noted that the metric has also been evaluated for each of the ship-specific estimates, which means that six values of the error measure are given for each of the realisations for any sub-scenario. Specifically, this means that the index $i$ takes "values" $i=\{$ Ro-Ro,R/V,FPSO,equal,heave,pitch $\}$. If one of the measures, take $\varepsilon_{\text {equal }}$, is selected as base-reference it can be readily visualised which of the six spectrum-estimates that yields the best (relative) agreement with the true generating wave spectrum by plotting $\varepsilon_{i}$ as function of $\varepsilon_{\text {equal }}$. The outcome is shown in Figure 9, and it is seen that linear fitting lines are associated to the respective results; pointing out that the slope of the linear fit is a direct measure of the agreement. It is found that among all six types of spectrum estimates (ship-specific as well as weighted), the best agreement is achieved from the pitch-based estimate, since it has the smallest slope $(=0.86)$.

\subsubsection{Integrated wave parameters}

The difference-metric $\varepsilon_{i}$ is useful as a comparative measure of the relative agreement among the various types of estimates. To supplement the comparative study between 

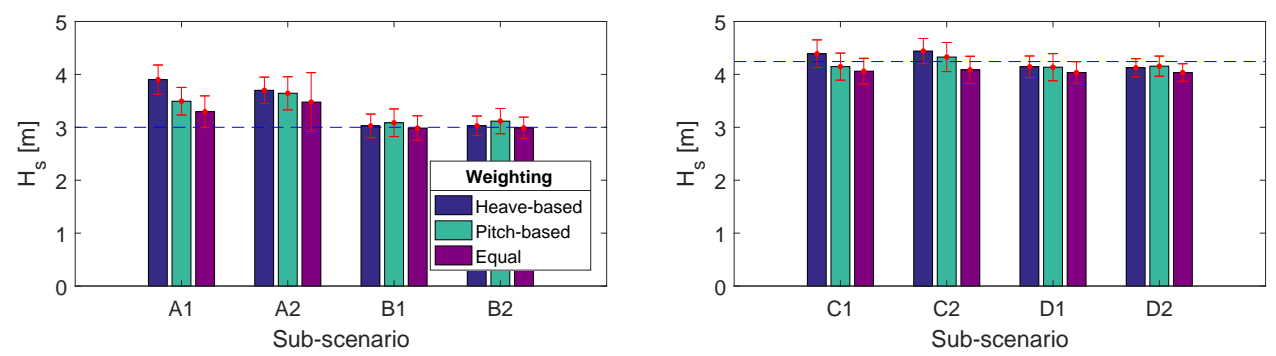

Figure 10: Statistics of estimates of the significant wave height. The true value is indicated by the dashed blue line, and the red arrow markers show plus/minus the standard deviation.
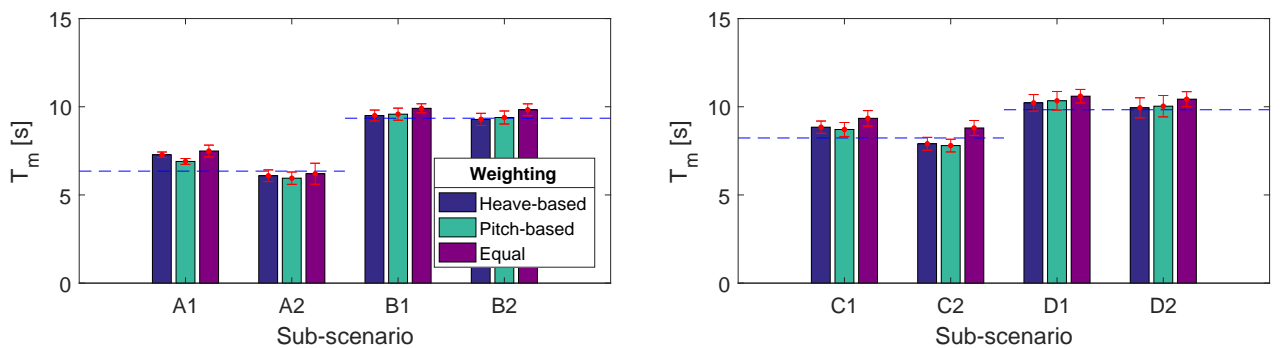

Figure 11: Statistics of estimates of the mean wave period.
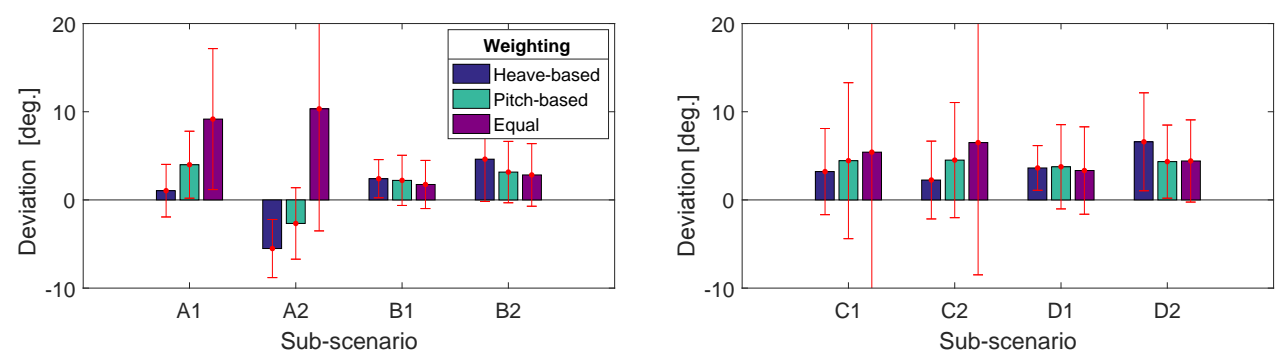

Figure 12: Statistics of estimates of the mean wave direction. The true value is, per definition, zero.

the different weighted estimates, the calculation of integrated wave parameters, or sea state parameters, such as significant wave height $H_{s}$, mean wave period $T_{m}$, and mean wave direction $\vartheta$ can be used. Consequently, Figures 10-12 show the statistics of the sea state parameters; leaving out the results of the ship-specific estimates. The statistics 
have been computed from the twenty realisations (i.e. spectrum estimates) of a given sub-scenario, and, in the plots, the height of the bars indicates the mean value, while the 'error markers' indicate plus/minus the standard deviation. The statistics of $H_{s}$ and $T_{m}$ are based on values obtained from the spectral moments $m_{n}$ of the weighted wave spectrum estimate, i.e.

$$
\begin{array}{rr}
H_{s, i}=4 \sqrt{m_{0, i}} & \text { Significant wave height } \\
T_{m, i}=2 \pi \frac{m_{0, i}}{m_{1, i}} & \text { Mean wave period } \\
m_{n, i}=\int_{0}^{\infty} \omega_{0}^{n} S_{i}^{0}\left(\omega_{0}\right) d \omega_{e} & n \text {-th order spectral moment }
\end{array}
$$

Note that the statistics of the wave direction estimate are in absolute values.

Generally, it can be seen from Figures 10-12 that the sea state parameters of the weighted wave spectrum estimates agree well with the true parameters, and, with no surprise (cf. Figure 9), it is found that, overall, the heave- and pitch-based weighted results have the best agreement. With a special focus on the individual wave parameters, the most noteworthy observations are the following: Significant wave height; the poorest estimates are obtained for sub-scenarios A1 and A2. This is explained because the wave systems of these sub-scenarios have most of the wave energy distributed at the higher frequencies and, hence, makes the sub-scenarios "susceptible" to the negative effects of the ships' filtering characteristics. Mean wave period; the estimates have accurate mean and small standard deviation in all cases. Wave direction; the mean values, i.e. the heights of the bars, are reasonable but large(r) standard deviations are observed, notably for some of the equally-weighted results. The reason for this is because of "outliers" obtained by the Ro-Ro and the FPSO vessels, especially in conditions where the wave system has energy distributed at the higher frequencies, which is the case for sub-scenarios $\mathrm{A} 1$ and $\mathrm{A} 2$, and $\mathrm{C} 1$ and $\mathrm{C} 2$, respectively.

The visualised comparisons (Figs. 10-12) of sea state parameters have been quantified in Table 3, showing the deviations of the estimated sea state parameters and the generating wave parameters. The deviations in $H_{s}$ and $T_{m}$, respectively, are relative to the true values, whereas the deviation in wave direction is the absolute number (in degrees). The table also includes the result for the peak wave period $T_{p}$ with deviations for both the swell and wind sea parts. Sub-scenarios A and B are represented by one-peaked wave 
Table 3: Deviation between estimates and true values of sea state parameters, dependent on how weighting is introduced. Here, the results are based on the transfer functions of heave $(\mathbf{H})$, pitch $(\mathrm{P})$, or an equal weighting $(\mathrm{E})$.

\begin{tabular}{|c|c|c|c|c|c|c|c|c|c|c|c|c|}
\hline & \multicolumn{3}{|c|}{ Dev. $H_{s}[\%]$} & \multicolumn{3}{|c|}{ Dev. $T_{m}[\%]$} & \multicolumn{3}{|c|}{ Dev. $T_{P}[\%]^{*}$} & \multicolumn{3}{|c|}{ Dev. $\vartheta$ [deg.] } \\
\hline & $\mathrm{H}$ & $\mathrm{P}$ & $E$ & $\mathrm{H}$ & $\mathrm{P}$ & $E$ & $\mathrm{H}$ & $\mathrm{P}$ & E & $\mathrm{H}$ & $\mathrm{P}$ & $\mathrm{E}$ \\
\hline A1 & 30 & 16 & 10 & 15 & 9 & 18 & $2 /-$ & $-2 /-$ & $3 /-$ & 1 & 4 & 9 \\
\hline A2 & 23 & 21 & 16 & -4 & -6 & -2 & $-1 /-$ & $1 /-$ & $-1 /-$ & -6 & -3 & 10 \\
\hline B1 & 1 & 3 & 0 & 2 & 2 & 6 & $0 /-$ & $0 /-$ & $1 /-$ & 2 & 2 & 2 \\
\hline B2 & 1 & 4 & 0 & 1 & 0 & 5 & $-2 /-$ & $-1 /-$ & $-1 /-$ & 5 & 3 & 3 \\
\hline $\mathrm{C} 1$ & 3 & -2 & -4 & 7 & 6 & 14 & $-17 / 5$ & $-9 / 13$ & $-13 / 10$ & 3 & 4 & 5 \\
\hline $\mathrm{C} 2$ & 5 & 2 & -4 & -4 & -5 & 7 & $-15 / 4$ & $-10 / 7$ & $-14 / 0$ & 2 & 5 & 7 \\
\hline D1 & -2 & -2 & -5 & 4 & 5 & 8 & $-18 /-6$ & $-10 /-4$ & $-18 /-10$ & 4 & 4 & 3 \\
\hline D2 & -3 & -2 & -5 & 1 & 2 & 6 & $-14 / 0$ & $-9 /-1$ & $-14 /-7$ & 7 & 4 & 4 \\
\hline
\end{tabular}

*swell period/wind sea period

spectra, and the estimates also "produce" just one peak (with high accuracy) for theses scenarios. The central finding from Table 3, including Figures 10-12, is that, while it does not make much difference for the total energy content (represented by $H_{s}$ ) whether the one or the other weighting is used, the energy distribution (represented by $T_{m}, T_{p}, \vartheta$ ) is better using the heave- or pitch-weighted estimates. And, although not shown/discussed, the general result reflected by Table 3 represents a substantial improvement of any of the ship-specific estimate.

\subsection{Comments about the uncertainty measure}

The case study has been considering a somewhat arbitrarily selected group of vessels; noting, however, that the ships are representing different filtering characteristics to better illustrate the idea in making SSE from multiple vessels. It should be interesting to consider other types of vessels to look at the results' sensitivity in this respect. Similarly, various weighting functions can be established; either with the purpose to be multiplied directly with the actual ship-specific wave spectrum estimates, like suggested in this study, or the weighting might be introduced with respect to a "post-processed calculation" of integrated wave parameters. In any case, it will be relevant to associate some sort of uncertainty to the estimate(s). In the present work, one practical measure has been suggested, see Subsection 4.3, and its outcomes for the considered sub-scenarios 


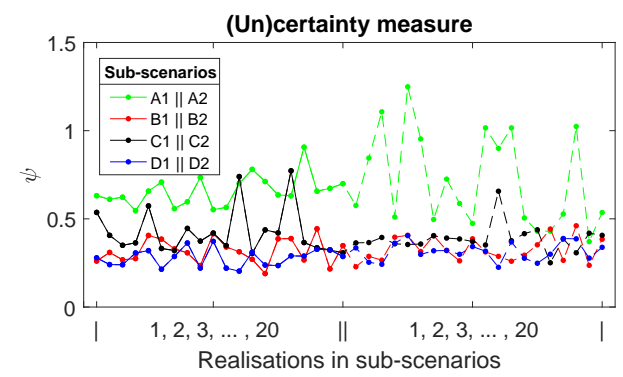

Figure 13: The measure based on Eq. (12) can be used as an indicator of the (un)certainty related to the wave spectrum estimate produced from multiple ship-specific estimates. A large number reflects large variation, i.e. uncertainty, among the particular ship-specific estimates in a given case.

are shown in Figure 13. Herein, the (un)certainty measure is based on the frequencywise variation among the individual ship-specific wave spectrum estimates, cf. Eq. (12). Inspection of Figure 13 reveals that large variations occur between the ship-specific estimates of sub-scenarios A1 and A2, whereas sub-scenarios B1, B2, D1, and D2 have small(er) uncertainty associated. It is evident from the figure that the most "problematic" sub-scenarios are A1, A2, C1, and C2; which were the same scenarios that generally reflected the least good agreement with the true generating wave spectrum. It needs to be kept in mind, however, that the (un)certainty measure (Eq. 12) is a relative number and, as such, cannot be used to directly infer whether any particular sea state estimate is good or bad. On the other hand, it should be interesting to apply/associate the measure in larger-scale studies where different subsets of a group of several vessels are used in network-based wave estimation. In such a case, it should be viable/feasible to select the final wave spectrum estimate from the subset of vessels associated with the smallest value of the uncertainty measure.

\section{Concluding remarks and future works}

The article has investigated the possibility to make sea state estimation using data from multiple ocean vessels simultaneously. The use of a single vessel as a (sailing) wave buoy is not new, but the simultaneous use of a group of vessels is. Herein, it becomes relevant to introduce weighting of the single (ship-specific) wave spectrum estimates, and 
the article has discussed one practical approach for this. Thus, it has been proposed to establish weight functions based on the sets of motion transfer functions of the particular vessels. The proposal does not include weighting based on the relative distance between the vessels, although this might be relevant in future studies.

The possession of simultaneous wave spectrum estimates from multiple ships facilitates an associated (un)certainty measure. The article has discussed one possible measure based on the frequency-wise variation of the individual ship-specific wave spectrum estimates.

The article contains a case study focused on numerical simulations of motion measurements from three vessels operating in a given confined ocean area. In the case study, it is shown that the (final) wave spectrum estimate can be improved by considering data from multiple ships simultaneously. In particular, it is interesting to note the capability to estimate (also) the higher-frequency wave components, if vessels of different dimensions are considered; thus observing a "reduced" effect of wave filtering.

The proposed network-based approach for sea state estimation requires further studies. One study should look into exactly what data to share and communicate, since the amount of data to be handled and exchanged from and in between the single vessels could be extensive; emphasising the need to ensure efficient data exchange among many (different) observation platforms. This topic has not been addressed, but it will be necessary to consider in future works.

Another future study related to the use of ships as wave buoys should introduce the idea to make sea state estimation entirely based on measurements data and associated analytics, and simply "discarding" the need for transfer functions in real(-time) in-service scenarios. In this context, it is understood that vast amounts of wave-induced response data may be generated and/or measured in conditions where the generating wave system is exactly known; for example as is the situation in simulation studies of time history recordings, cf. Section 5. Subsequently, the generated (or measured) response recordings could be analysed and cast through machine learning procedures with the purpose to train algorithms to find optimum wave spectrum estimates under given conditions. The learning procedures may follow different approaches from more simple analytical models to deep learning algorithms for image recognition. 
On a higher level, the use of multiple ships for sea state estimation could be a first step towards sea state estimation using a heterogenous network of (wave) observation platforms, where other observation platforms such as classical wave buoys, wave radar, and satellites are also relevant means to consider.

\section{Acknowledgement}

The authors would like to thank Prof. emeritus Jørgen Juncher Jensen for valuable discussions and input. This work has been supported by the Research Council of Norway through the Centres of Excellence funding scheme, Project number 223254-AMOS.

\section{References}

[1] I. Utne, A. Sørensen, I. Schjølberg, Risk Management of Autonomous Marine Systems and Operations, in: Proc. 36th OMAE, Trondheim, Norway, 2017.

[2] M. Ludvigsen, A. Sørensen, Towards integrated autonomous underwater operations for ocean mapping and monitoring, Annual Reviews in Control 42 (2016) 145-157.

[3] S. Barstow, J.-R. Bidlot, S. Caires, M. Donelan, W. Drennan, H. Dupuis, H. Graber, J. Green, O. Gronlie, C. Gurin, Measuring and Analysing the Directional Spectrum of Ocean Waves, COST Office, 2005.

[4] M. Tucker, T. Pitt, Waves in Ocean Engineering, Vol. 5 of Elsevier Ocean Engineering Book Series, Elsevier, 2001.

[5] S. Graham, Remote Sensing, https://earthobservatory.nasa.gov/Features/RemoteSensing/, 1999 (Accessed: 2018-05-10).

[6] M. Tucker, Recommended standard for wave data sampling and near-real-time processing, Ocean Engineering 20 (1993) 459-474.

[7] J. Nieto-Borge, K. Reichert, J. Dittmer, Use of nautical radar as a wave monitoring instrument, Coastal Engineering 37 (1999) 331-342.

[8] U. D. Nielsen, Estimations of on-site directional wave spectra from measured ship responses, Marine Structures 19 (2006) 33-69.

[9] U. D. Nielsen, Introducing two hyperparameters in Bayesian estimation of wave spectra, Probabilistic Engineering Mechanics 23 (2008) 84-94.

[10] U. D. Nielsen, D. C. Stredulinksy, Sea state estimation from an advancing ship - A comparative study using sea trial data, Applied Ocean Research 34 (2012) 33-44.

[11] A. H. Brodtkorb, U. D. Nielsen, A. J. Sørensen, Sea State Estimation Using Vessel Response in Dynamic Positioning, Applied Ocean Research 70 (2018) 76-86.

[12] U. D. Nielsen, A. H. Brodtkorb, A. J. Sørensen, A brute-force spectral approach for wave estimation using measured vessel motions, Marine Structures 60 (2018) 101-121. 
[13] T. Iseki, K. Ohtsu, Bayesian estimation of directional wave spectra based on ship motions, Control Engineering Practice 8 (2000) 215-219.

[14] T. Iseki, D. Terada, Bayesian Estimation of Directional Wave Spectra for Ship Guidance Systems, International Journal of Offshore and Polar Engineering 12 (2002) 25-30.

[15] E. A. Tannuri, J. V. Sparano, A. N. Simos, J. J. D. Cruz, Estimating directional wave spectrum based on stationary ship motion measurements, Applied Ocean Research 25 (2003) 243-261.

[16] R. Pascoal, C. G. Soares, A. J. Sørensen, Ocean Wave Spectral Estimation Using Vessel Wave Frequency Motions, Journal of Offshore Mechanics and Arctic Engineering 129 (2007) 90-96.

[17] A. N. Simos, J. V. Sparano, E. A. Tannuri, V. L. F. Matos, Directional Wave Spectrum Estimation Based on a Vessel 1st Order Motions: Field Results, in: Proc. of 17th International Offshore and Polar Engineering Conference, Lisbon, Portugal, 2007.

[18] J. V. Sparano, E. A. Tannuri, A. N. Simos, V. L. F. Matos, On the Estimation of Directional Wave Spectrum Based on Stationary vessels 1st Order Motions: A New Set of Experimental Results, in: Proc. of OMAE'08, Lisbon, Portugal, 2008.

[19] R. Pascoal, C. G. Soares, Kalman filtering of vessel motions for ocean wave directional spectrum estimation, Ocean Engineering 36 (2009) 477-488.

[20] R. Pascoal, L. P. Perera, C. G. Soares, Estimation of Directional Sea Spectra from Ship Motions in Sea Trials, Ocean Engineering 132 (2017) 126-137.

[21] F. L. de Souza, E. Tannuri, P. cardozo de Mello, G. Franzini, J. Mas-Soler, A. Simos, Bayesian estimation of directional wave-spectrum using vessel movements and wave-probes: Proposal and preliminary experimental validation, in: Proceedings of 36th OMAE, Trondheim, Norway, 2017.

[22] I. A. Milne, M. Zed, Full-scale validation of the hydrodynamic motions of a ship derived from a numerical hindcast, Ocean Engineering (accepted for publication).

[23] U. D. Nielsen, A concise account of techniques available for shipboard sea state estimation, Ocean Engineering 129 (2017) 352-362.

[24] G. Lindgren, I. Rychlik, M. Prevosto, Stochastic Doppler shift and encountered wave period distributions in Gaussian waves, Ocean Engineering 26 (1999) 507-518.

[25] U. D. Nielsen, Transformation of a wave energy spectrum from encounter to absolute domain when observing from an advancing ship, Applied Ocean Research 69 (2017) 160-172.

[26] U. D. Nielsen, Deriving the absolute wave spectrum from an encountered distribution of wave energy spectral densities, Ocean Engineering 165 (2018) 194-208.

[27] J. Nielsen, N. Pedersen, J. Michelsen, U. Nielsen, J. Baatrup, J. Jensen, E. Petersen, SeaSense Real-time Onboard Decision Support, in: Proc. of WMTC2006, London, UK, 2006.

[28] U. D. Nielsen, Response-based estimation of sea state parameters - influence of filtering, Ocean Engineering 34 (2007) 1797-1810.

[29] U. D. Nielsen, The wave buoy analogy - estimating high-frequency wave excitations, Applied Ocean Research 30 (2008) 100-106.

[30] E. Tu, G. Zhang, L. Rachmawati, E. Rajabally, G.-B. Huang, Exploiting AIS Data for Intelligent Maritime Navigation: A Comprehensive Survey, IEEE Transactions on Intelligent Transportation 
Systems 19 (2018) 1559-1582.

[31] R. Bhattacharyya, Dynamics of Marine Vehicles, John Wiley \& Sons, 1978.

[32] R. Beck, W. Cummins, J. Dalzell, P. Mandel, W. Webster, Vol. III: Motions in Waves and Controllability, in: E. Lewis (Ed.), Principles of Naval Architecture, Second Revision, SNAME, 1989, pp. $1-188$.

[33] J. Journée, W. Massie, Offshore Hydromechanics, lecture notes in course offered at TU Delft (January 2001)

[34] N. Montazeri, J. J. Jensen, U. D. Nielsen, Uncertainties in ship-based estimation of waves and responses, in: Proc. of MTS/IEEE OCEANS15, Washington, DC, USA, 2015.

[35] A. H. Brodtkorb, U. D. Nielsen, A. J. Sørensen, Online wave estimation using vessel motion measurements, in: Proc. of 11th IFAC Conf. CAMS, Opatija, Croatia, 2018.

[36] U. D. Nielsen, A. H. Brodtkorb, Ship motion-based wave estimation using a spectral residualcalculation, in: Proc. of MTS/IEEE OCEANS18, Kobe, Japan, 2018. 